\title{
Review: \\ Philipp Otto and Eike Gräf (eds.): 3TH1CS: A Reinvention of Ethics in the Digital Age?
}

\author{
Reviewed by Dr. Markus Haag \\ Visiting Lecturer at the University of Bedfordshire, UK
}

The sub-title of "3TH1CS: A Reinvention of Ethics in the Digital Age?" ends with a question mark, thus setting the scene for an exploration of a diverse range of issues and challenges that are currently arising in relation to the ubiquity of data and how this impacts on businesses, individuals and society. Currently, there seems to be quite an intense coverage not only in academic quarters around digital ethics, particularly on artificial intelligence (AI) but also in the wider population. Businesses and individuals seem to be more aware than ever of not only the (potential) benefits of the digital age to them, but also of the ethical challenges that this presents.

It is therefore to be very much welcomed to have this edited book covering a broad range of issues, for example: artificial agents, sex robots, fake news, machine learning, drones, healthcare services, autonomous vehicles, games, law enforcement, gender, data protection, and others. This is covered in 21 separate chapters, so it does not seem sensible to review a large number of these chapters, and I will therefore present my own personal views on the general overall 'tone' of the book. It also benefits from a wide range of backgrounds and experiences of the contributing authors, who come from the USA, Germany, Italy, the UK, China, and elsewhere.

3TH1CS seems to suggest that there needs to be a reinvention of ethics in the digital age, at least to some degree. Most of the chapters focus more on highlighting issues and raising questions that businesses and individuals need to address to solve ethical dilemmas rather than providing answers. This is not a criticism, as discussions around this have only relatively recently been held on a broader and more intensive scale. This is supported in the introductory chapter by the editors when they state - and it is worth quoting here more fully: "We have to define very specific contexts to morally evaluate actions, procedures, applications, organizations and systems and to come up with ethically desirable ways to shape them. [...] We need to fully understand what we are facing before we can take a moral stance towards an issue. We hope that this book will be useful in getting there." (p. 8).

In my opinion, this is clearly the case. I found the chapter that transcribes an interview with Prof Rafael Capurro quite useful to set the scene, as he comments on a range of concrete application areas rather than remaining vague. The holy grail of artificial intelligence, general AI, seems to be outside of this need for having to define very specific contexts to evaluate ethical approaches - so if you expect a book on general AI, this is not for you.

I was also pleased that there is at least some coverage of the ethical implications of the General Data Protection Regulation (GDPR) which will come into force in May 2018. This has wide ranging consequences not only for businesses but also individuals and I do not think that individuals are yet aware of this sufficiently.

I felt that the range of topics was particularly useful for people with some previous background around areas such as digital ethics, information ethics, or similar. However, I think the book misses a trick in terms of marketing this book to a wider audience. Maybe for a second edition, the chapters could contain some additional help for the reader to navigate through the huge range of ideas presented. Depending on the nature and complexity of these ideas, this could be done in table format, through figures, or (and I personally think this would work particularly well) through chapter summaries that also contain references to related reading materials. 
Overall, the stances expressed in the book seemed relatively neutral in whether an increased prevalence of data in the near future is good or bad. It seemed to say: well, humans are likely to have a good level of control over whether the consequences are good or bad, as long as there is transparency and an open discussion around ethical aspects surrounding data.

To sum up, this is a welcome and timely discussion on most subject areas related to digital ethics for readers with some prior knowledge of the field. The last words of the edited chapters in this book are "In any given instance we need to consider the chain of causality and the most effective ways to prevent harm." (p. 210). For me, this seems an appropriate way to wrap up the book (even though it was probably not intended that way), reminding readers to analyse the specific situation first and then act with the key aim of preventing harm to individuals whenever businesses and individuals use digital technologies.

It will be fascinating to revisit the book in, say, 2027, and see to which degree there is then an answer to the question in the title, and more importantly, what the different answers (in the plural) are - relevant to the specific contexts. It is to be hoped that the digital age of the future will be beneficial to individuals and society. 